Wilfrid Laurier University

Scholars Commons @ Laurier

Physics and Computer Science Faculty

Publications

Physics and Computer Science

4-1993

\title{
Mode Transforming Properties of Tapered Single-Mode Fiber Microlenses
}

Chris W. Barnard

University of Ottawa

John W.Y. Lit

Wilfrid Laurier University, jlit@wlu.ca

Follow this and additional works at: https://scholars.wlu.ca/phys_faculty

\section{Recommended Citation}

Barnard, Chris W. and Lit, John W.Y., "Mode Transforming Properties of Tapered Single-Mode Fiber Microlenses" (1993). Physics and Computer Science Faculty Publications. 23.

https://scholars.wlu.ca/phys_faculty/23

This Article is brought to you for free and open access by the Physics and Computer Science at Scholars Commons @ Laurier. It has been accepted for inclusion in Physics and Computer Science Faculty Publications by an authorized administrator of Scholars Commons @ Laurier. For more information, please contact scholarscommons@wlu.ca. 


\title{
Mode transforming properties of tapered single-mode fiber microlenses
}

\author{
Chris W. Barnard and John W. Y. Lit
}

\begin{abstract}
The Gaussian approximation that is typically used to estimate single-mode fiber microlens performance is investigated. It is applied to hemispheric lenses on two types of tapered single-mode fiber. Theoretical and experimental results are compared. The first type of taper, which is fabricated by pulling a fiber while it is melted, has a tapered core and a tapered cladding. The second type of taper, which is fabricated by etching the cladding, has a tapered cladding only. For a tapered-core fiber, coupling to the cladding-guided modes and the finite radius of curvature of the wave front before the lens must be considered to predict the lens spot size accurately, whereas the spot size of a tapered-cladding lens can be predicted from the lens diameter alone. Thus the spot size of a lens on a tapered-cladding fiber is easier to predict and control than that of a lens on a tapered-core fiber. It is also shown that the usual theory used to predict the spot size gives accepted values for tapered-cladding lenses but not for tapered-core lenses.
\end{abstract}

Key words: Fiber microlenses, single-mode fiber coupling.

\section{Introduction}

Several methods have been used to fabricate a microlens on a single-mode fiber to increase the coupling between a fiber and a laser diode ${ }^{1}$ or an optical waveguide device. $^{2}$ Typically the lens is located at the end of a taper to decrease the lens diameter and, hence, decrease its spot size. ${ }^{1}$ If the taper is fabricated by pulling and melting, ${ }^{3}$ a tapered-core lens results, as shown in Fig. 1(a). Alternatively, fibers may be tapered by acid etching, which results in a tapered-cladding lens, ${ }^{4}$ as shown in Fig. 1(b). To control tapered fiber microlenses, we must better understand the dependence of the spot size on the taper and lens dimensions. Several authors (e.g., Ref. 3) have estimated the spot size of a tapered-core lens by first calculating the approximate Gaussianmode radius at the lens and by using Gaussian optics, assuming that the radius of curvature of the wave front at the lens is infinite. The results of this

When this work was performed the authors were with the Guelph Waterloo Program for Graduate Work in Physics, University of Waterloo, Ontario, N2L 3G1, Canada; C. Barnard is now with the Department of Electrical Engineering, University of Ottawa, Ottawa, Ontario, K1N 6N5, Canada. J. Lit is with the Department of Physics and Computing, Wilfrid Laurier University, Waterloo, Ontario, N2L 3C5, Canada.

Received 11 October 1991.

0003-6935/93/122090-05\$05.00/0.

(C) 1993 Optical Society of America. analysis have not agreed well with the experimental results ${ }^{3}$ for two reasons. First, coupling from the core-guided mode to the cladding-guided modes has been neglected. Second, the rate of expansion and contraction of the mode caused by the tapered core can be significant, rendering invalid the assumption that the mode is propagating as a plane wave before the lens.

Here we investigate how the tapering and the lens diameter affect the spot sizes of the two types of taper lens. The analysis accounts for the coupling from the core-guided mode to the fundamental claddingguided mode, and also examines how the finite radius of curvature of the wave front affects the focusing properties. We first describe the focusing effect of a spherical refracting surface on a Gaussian beam, and then apply the result to the two types of microlens. We use the local mode theory, ${ }^{5}$ which assumes that the mode in a fiber taper at any point along the taper is equal to the equivalent mode of a straight fiber with the same core diameter. This requires a gradual, adiabatic taper. ${ }^{6}$ The lens spot sizes are calculated by assuming that the wave front has an infinite radius of curvature at the lens entrance face. The validity of this assumption is tested by using a more rigorous analysis for a typical tapered-core lens. The example shows that the rate of expansion and contraction of the mode in the taper, which depends on the taper angle, can significantly affect the lens spot size. We consequently develop an estimate of 


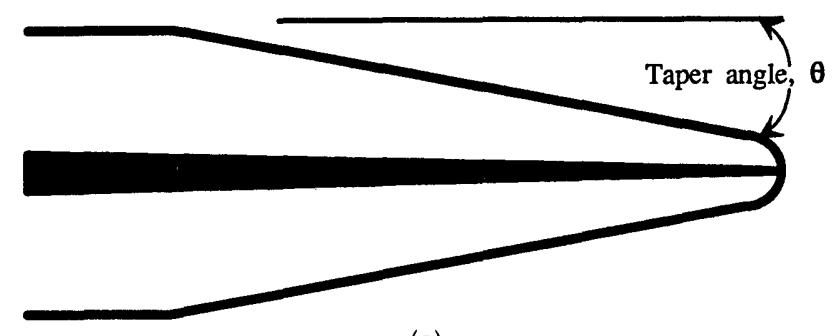

(a)

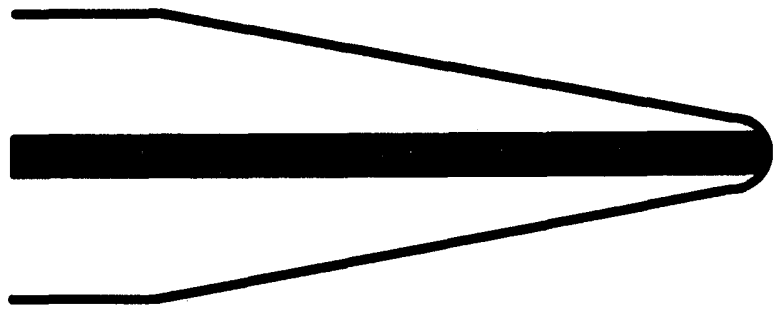

(b)

Fig. 1. (a) Tapered-core lens; (b) tapered-cladding lens.

the spot size that depends on the taper angle as well as on the lens diameter.

To test the theory, we present measurements of the spot size as a function of the lens radius for lenses on both types of taper. The analysis uses the parameters of the experimental fibers. These fibers, which are single moded at $0.633 \mu \mathrm{m}$, have core and cladding refractive indices of 1.4575 and 1.453 , respectively, and core and cladding diameters equal to 4.1 and 125 $\mu \mathrm{m}$, respectively. By comparing the theoretical and the experimental results, we show that, as expected, the simple model that assumes an infinite radius of curvature can accurately predict the spot size of a tapered-cladding lens but it cannot accurately predict the spot size of a tapered-core lens.

\section{Taper Lens Mode Transforming Properties}

A. Focusing of a Gaussian Beam by a Spherical Surface

A Gaussian approximation is typically used $^{1}$ to predict the focusing effect of a fiber microlens. ${ }^{3} \mathrm{~A}$ Gaussian beam with radius $w_{L}$ and radius of curvature $R_{L}$ within a fiber of refractive index $n$ is focused by a lens with radius $r$ to a spot size in air, which is given by

$$
w_{f}^{2}=\frac{w_{L}^{2}}{1+\left(\frac{\pi w_{L}^{2}}{\lambda}\right)^{2}\left(\frac{n-1}{r}-\frac{n}{R_{L}}\right)^{2}},
$$

where $\lambda$ is the free-space wavelength. Since the wave front in an untapered fiber has an infinite radius of curvature, the expression for the spot size of a lens on an untapered fiber reduces to

$$
w_{f}^{2}=\frac{w_{L}^{2}}{1+\left[\frac{\pi w(n-1)_{L}^{2}}{r \lambda}\right]^{2}} .
$$

If the incident fiber mode is expanding or contracting because of tapering, $R_{L}$ will be finite, and Eq. (1) should be used to estimate the spot size accurately, unless

$$
\left|R_{L}\right| \gg \frac{r n}{n-1} .
$$

Note that, for the same incident mode radius, an incident plane wave has a larger spot size than a contracting beam $\left(R_{L}<0\right)$ and a smaller spot size than an expanding beam $\left(R_{L}>0\right)$. The spot size can be estimated from Eq. (2) if the lens and mode radii are known. Marcuse's estimate for the mode radius is typically used ${ }^{7}$ :

$$
\frac{w_{L}}{a}=0.65+\frac{1.619}{V^{3 / 2}}+\frac{2.879}{V^{6}}
$$

where $a$ is the core radius and the $V$ number is given by

$$
V=\frac{2 \pi a}{\lambda}\left(n_{1}^{2}-n_{2}^{2}\right)^{1 / 2},
$$

where $n_{1}$ and $n_{2}$ are the core and the cladding refractive indices, respectively. The estimate in Eq. (2) has been used by various authors ${ }^{3,4}$ to calculate the spot size of lenses on both tapered-cladding and tapered-core fibers. In this paper we investigate the validity of this estimation for lenses on the two types of fiber and demonstrate that it is accurate only for tapered-cladding fibers.

\section{B. Mode Transformation in a Tapered-Core Fiber}

For a drawn taper [Fig. 1(a)] it is assumed that the cladding and the core radii maintain their initial ratio. $^{3}$ As the core-guided mode propagates along the taper it first contracts slightly, then spreads into the cladding, and gradually couples to the claddingquided modes. Coupling will then occur among the cladding-guided modes. ${ }^{8}$ For simplicity we assume that coupling occurs only from the core-guided mode to the fundamental cladding-guided mode. To calculate the mode radius at a given point on the taper, we calculate the fundamental cladding-guided mode radius, compare this with the core-guided mode radius, and choose the smaller of the two as the mode radius. This is equivalent to assuming that the core-guided mode couples completely to the fundamental claddingguided mode at the point at which the two mode radii are equal. (The result, based no this assumption, will be an estimate, since $100 \%$ mode coupling does not occur.) The $V$ for the cladding-guided mode is calculated by substituting into Eq. (5) the cladding index for $n_{1}, 1$ (the refractive index of air) for $n_{2}$, and the cladding radius for $a$. The core- and the claddingguided mode radii at any point on the taper can thus be estimated from the fiber cladding diameter if the numerical aperture is known.

To illustrate the mode transformation, we use as an example a linear 600- $\mu \mathrm{m}$-long taper (which corresponds to a taper angle of $5.9^{\circ}$ ). Figure 2 shows the 


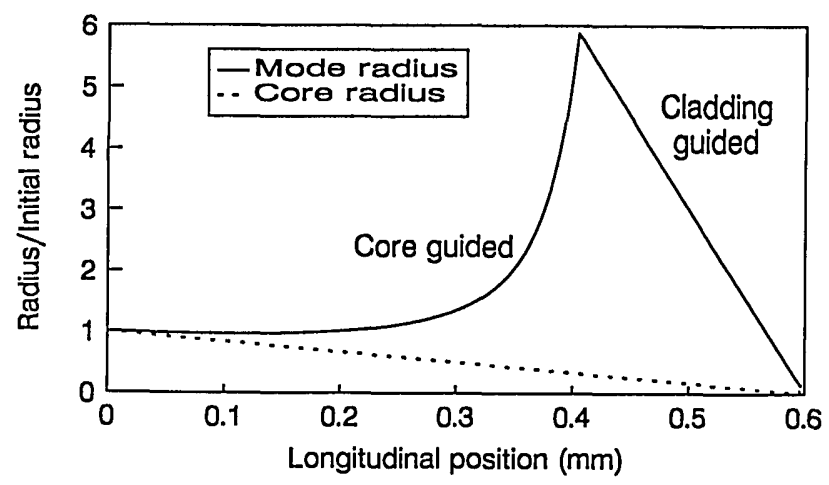

Fig. 2. Mode transformation in a 600- $\mu \mathrm{m}$-long tapered-core fiber.

tapering of the core radius (dashed curve) and the calculated mode radius (solid curve) in the taper. As discussed above, the mode radius first decreases slightly, and then increases along the taper as the mode spreads into the cladding. After coupling to the fundamental cladding-guided mode, the mode radius decreases monotonically as the light radiates out of the fiber.

To calculate the focusing effects of a lens on the taper we assume that the lens is spherical with a diameter equal to the tapered fiber diameter just before the lens. We also neglect the contraction or expansion of the mode so that Eq. (2) can be used. The validity of this assumption is discussed below. The dashed curve in Fig. 3 shows the ratio of spot size radius $w_{f}$ to the initial mode radius (mode radius in an untapered fiber) as a function of the normalized lens radius. The rightmost point of the plateau of the curve indicates where the core-guided mode couples to the cladding-guided mode.

For the fundamental cladding-guided mode, because both $V$ and $w_{L}$ are large, Eq. (2) reduces to

$$
w_{f} \approx \frac{\lambda f}{\pi w_{L}} \approx \frac{\lambda}{0.65 \pi(n-1)} .
$$

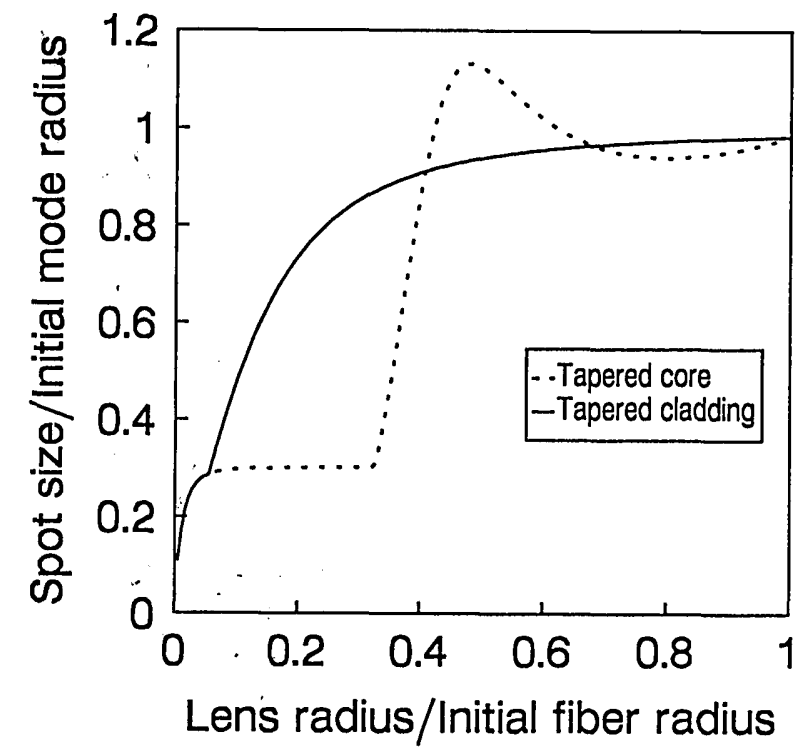

Fig. 3. Theoretical spot size versus taper lens radius.
Thus, in the cladding-guiding section of the taper, when $\pi w_{L}{ }^{2} / \lambda \gg r /(n-1)$, the spot size is independent of the lens radius. For our fibers this means that $w_{f}$ is almost constant at 0.3 times the original mode radius; this explains the plateau of the curve in Fig. 3. This result is advantageous in that this type of tapered lens will have a predictable spot size that is insensitive to the lens radius. However, as we now show, the spot size depends strongly on the taper angle.

To determine the effect of the taper angle we note that the radius of curvature of a Gaussian beam can be expressed as

$$
R_{L}=\frac{w}{\mathrm{~d} w / \mathrm{d} z} \approx \frac{r}{\mathrm{~d} r / \mathrm{d} z}=\frac{r}{\tan \theta},
$$

where $z$ is the direction of propagation." The rate of change of the mode radius in a taper can be determined from Eq. (4) and related to the taper angle $\theta$, where $\tan \theta=\mathrm{d} r / \mathrm{d} z$, and $r$ is the fiber radius. For $V \gg 1$, which is the case for the cladding-guided mode, the last two terms in Eq. (4) become much smaller than 0.65 , and we get the approximation in Eq. (7). Otherwise $\mathrm{d} w_{L} / \mathrm{d} z$ can be calculated numerically from Eqs. (4) and (5) for any point on a taper if its dimensions are known. Note that $\mathrm{d} w_{L} / \mathrm{d} z$ and hence $R_{L}$ are positive if the beam is expanding and negative if the beam is contracting. Once $R_{L}$ is found it can be substituted into Eq. (1) to achieve a more accurate estimate of the microlens spot size. Using Eq. (7) and the approximation in Eq. (3), we may neglect $R_{L}$ if $\tan \theta \ll 1-1 / n$ or $\theta \ll 17^{\circ}$ for fused-silica fibers. The typical taper anglès are $5-10^{\circ}$ for the drawn tapers, so neglecting the expansion or contraction of the incident mode can significantly affect the estimated spot size.

Figure 4 shows, for the taper in Fig. 2, the lens spot size as a function of its position on the taper and calculated with an estimate for $R_{L}$ (solid curve). It is compared with the one that is calculated by assuming that $R_{L}=\infty$ (dashed curve). $R_{L}$ is estimated at each point on the taper by using the first term on the right of Eq. (7). From Eqs. (4) and (5), $w_{L}$ is a function of

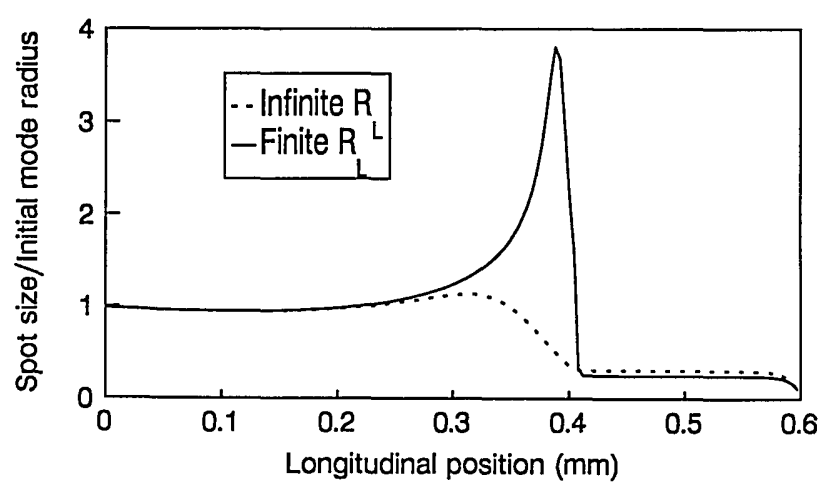

Fig. 4. Comparison of lens spot size versus position on the taper of Fig. 2 calculated (i) by estimating $R_{L}$ and (ii) by assuming that $R_{L}$ is infinite. 
core radius $a(z)$, and $\mathrm{d} w_{L} / \mathrm{d} z$ is a function of $\alpha(z)$ and $\mathrm{d} a / \mathrm{d} z$. Assuming that the core-to-cladding radius ratio, $a / r=\gamma$, is constant along the taper, $w_{L}$ and $\mathrm{d} w_{L} / \mathrm{d} z$ are found by using $a(z)=\gamma r(z)$ and $\mathrm{d} a / \mathrm{d} z=$ $\gamma \mathrm{d} r / \mathrm{d} z$. The solid curve, which accounts for the expansion and contraction of the beam, lies above the dashed curve when the beam is expanding and below the dashed curve when the beam is contracting. The curves indicate that the results obtained by assuming an infinite radius of curvature are approximate, at best.

The substitution of Eq. (7) into Eq. (1) gives the final waist radius as a function of the taper angle for the case in which the lens is placed at the beginning of the cladding-guided section (the plateau region of Fig. $3)$. For small taper angles, in this portion of the taper the second term in the denominator of Eq. (1) is much larger than 1 (the first term), which is therefore neglected. With $w_{L} \approx 0.65 r$, this leads to

$$
w_{f}=\left[\left(\frac{0.65 \pi}{\lambda}\right)|n-1-n \tan \theta|\right]^{-1} .
$$

The above gives an estimate for the spot size of the cladding-guided mode that depends on the taper angle only. According to approximation (8), which is valid for small angles only, as the taper angle increases the mode radius increases. Thus, to minimize the spot size, the tapering should be gradual before the lens. The deviation of the actual spot size from that estimated by assuming an incident plane wave increases as the taper angle increases. These lenses thus have the disadvantage that an accurate determination of the focusing effects of a lens requires a knowledge of the taper profile as well as of the lens radius, and the calculation can only be done numerically. ${ }^{9}$ The important result is that the spot size depends on the taper angle as well as on the lens radius for a lens formed on the section of a taper where the mode is contracting or expanding. The spot size can still be estimated if the taper profile before the lens is known and the taper angle is not too large.

\section{Mode Transformation in a Tapered-Cladding Fiber}

The analysis of mode transformation in a taperedcladding lens has been discussed in detail elsewhere. ${ }^{4}$ The spot size calculated from Eq. (2) is given by the solid curve in Fig. 3. ${ }^{4}$ The assumption of inequality (3) is better for this taper since the rate of contraction of the mode is much smaller than in a tapered-core fiber. Thus the approximation of Eq. (2) is expected to be much better for this type of lens.

\section{Experiment and Discussion}

The fabrication and the measurement of claddingtapered lenses was discussed in a previous paper. ${ }^{4}$ Tapered-core fibers are fabricated by melting and pulling suspended fibers with a coaxial $5-\mathrm{W} \mathrm{CO}_{2}$ laser.

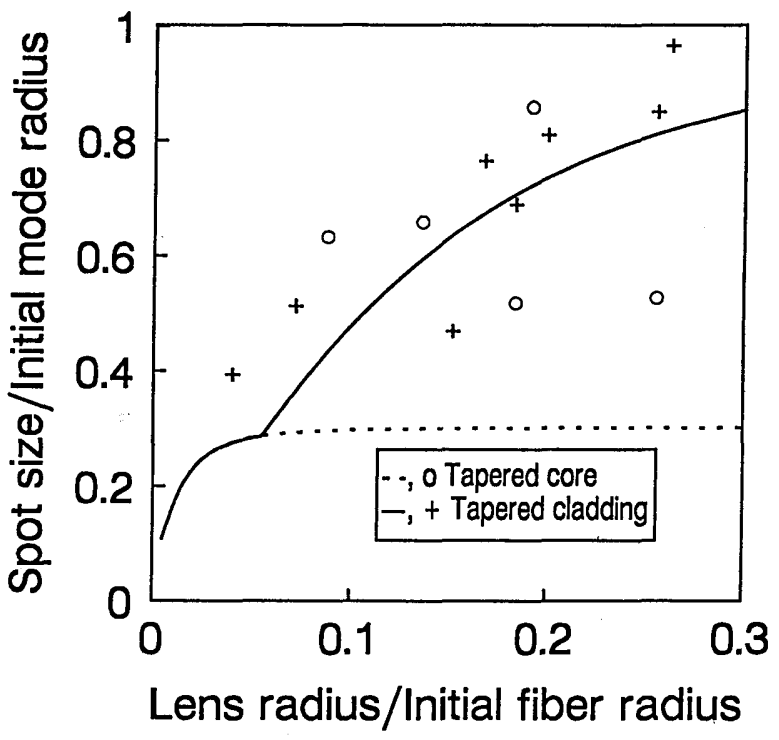

Fig. 5. Experimental points and theoretical curves of spot size versus lens radius.

Lenses are then formed by laser melting the ends of tapered fibers. ${ }^{4}$ Figure 5 shows the experimental spot-size measurements. The theoretical curves are taken from Fig. 3. This figure shows that the predicted spot sizes for the lenses on the etched tapers agree quite well with the measured values, while the experimental points for the tapered core lenses do not agree with the theory. We point out two factors to account for this phenomenon. The rate of contraction and expansion of the mode that is due to tapering, which results in a finite $R_{L}$, was neglected in calculating the curves of Fig. 5. The assumption that $100 \%$ coupling from the core-guided mode to the fundamental cladding-guided mode at the point at which their mode radii are equal is only an estimation of the complicated coupling that occurs between the core- and the cladding-guided modes. Except for small lens diameters, the two factors have little effect on the spot size of a tapered-cladding lens. Thus the spot size of a lens on a tapered-cladding fiber can be predicted from the lens diameter only. For a taperedcore lens the taper profile before the lens does seriously affect the latter factors. As a result, even if the lens radius can be accurately controlled, it is difficult to control a tapered-core lens spot size.

We compared the accuracy of predicting a fiber lens spot size from only the lens radius for two types of fiber lens. The approximation is valid for taperedcladding lenses but is invalid for tapered-core lenses because mode conversion and mode expansion are neglected. Predicting the tapered-core lens spot sizes requires the taper angle as well as the lens radius.

This work was supported by the Natural Sciences and Engineering Research Council of Canada. C. W. Barnard was supported by a Natural Sciences and Engineering Research Council of Canada Postgraduate Scholarship. 


\section{References}

1. H. Ghafoori-shiraz and T. Asano, "Microlens for coupling a semiconductor laser to a single-mode fiber," Opt. Lett. 11, 537-539 (1986).

2. Y. C. Chung, J. M. Weisenfeld, G. Raybon, U. Koren, and Y. Twu, "Intermodulation distortion in a multiple-quantum-well semiconductor optical amplifier," IEEE Photon. Tech. Lett. 3, 130-132 (1991).

3. R. Keil, E. Klement, K. Mathyssek, and J. Wittmann, "Experimental investigation of the beam spot size radius in single-mode fiber tapers," Electron. Lett. 20, 621-622 (1984).

4. C. W. Barnard and J. W. Y. Lit, "Single-mode fiber microlens with controllable spot size," Appl. Opt. 30, 1958-1962 (1991).
5. A. W. Snyder, and J. D. Love, Optical Waveguide Theory (Chapman \& Hall, New York, 1983), Chap. 19.

6. S. Lacroix, F. Gonthier, and J. Bures, "All-fiber wavelength filter from successive biconical tapers," Opt. Lett. 11, 671-673 (1986).

7. D. Marcuse, "Loss analysis of single-mode fiber splices," Bell Syst. Tech. J. 56, 703-718 (1977).

8. A. W. Snyder, "Coupling of modes on a tapered dielectric cylinder," IEEE Trans. Microwave Theory Tech. MTT-18, 383-392 (1970).

9. B. Hermansson, D. Yevick, and J. Saijonmaa, "Propagatingbeam-method analysis of two-dimensional microlenses and three-dimensional taper structures," J. Opt. Soc. Am. A 1, 663-671 (1984). 